\title{
Tendência da Energia Estática Global
}

\author{
Samyr Barata Chebly ${ }^{1}$ (D), Enilson Palmeira Cavalcanti ${ }^{1}$, Ranyére Silva Nóbrega ${ }^{2}$, \\ Magaly de Fátima Correia ${ }^{1}$ \\ ${ }^{1}$ Universidade Federal de Campina Grande, Campina Grande, PB, Brasil. \\ ${ }^{2}$ Departamento de Ciências Geográficas, Universidade Federal de Pernambuco, Recife, PE, \\ Brasil.
}

Recebido em: 5 de Fevereiro de 2020 - Aceito em: 17 de Maio de 2021

\begin{abstract}
Resumo
Neste estudo objetivou-se analisar tendências da energia estática da atmosfera para o Globo, por meio das reanálises do National Centers for Envionmental Prediction/National Center for Atmospheric Research (NCEP/NCAR), portanto foram analisadas as tendências das energias estáticas seca, úmida e saturada para o Hemisfério Norte (H.N.), Hemisfério Sul (H.S.) e Globo, durante 1987-2016. Para o cálculo das energias e integração vertical foram utilizados dados mensais das variáveis da pressão em superfície, temperatura do ar, umidade específica e altura geopotencial das reanálises do NCEP/NCAR e apresentados em formas de tabela, gráfico e imagens, como teste de significância estatística de Mann Kendall e $t$ de Student foram aplicados. Os resultados mostram que a variabilidade sazonal das energias estáticas para o H.N e H.S apresentam valores máximos (mínimos) na estação de verão (inverno). Tendências positivas são observadas nas séries da energia estática seca, úmida e saturadas, assim como os testes estatísticos indicam significância de $99 \%$ $(\alpha=0,01)$ para as tendências. Nas espacializações das tendências no Globo, verifica-se expressiva cobertura de áreas com tendências médias de $-0,5$ a $4,5 \times 10^{2}\left(\mathrm{~kJ} / \mathrm{m}^{2}\right) /$ ano.
\end{abstract}

Palavras-chave:: convecção, $t$ de Student, reanálises, energias estáticas.

\section{Global Static Energy Trend}

\begin{abstract}
This study aimed to analyze trends in static energy from the atmosphere to the Globe, through reanalysis from the National Centers for Envionmental Prediction / National Center for Atmospheric Research (NCEP / NCAR), so the trends in dry, wet and dry static energies were analyzed. saturated for the Northern Hemisphere (HN), Southern Hemisphere (HS) and Globo, during 1987-2016. For the calculation of energies and vertical integration, monthly data of the variables of surface pressure, air temperature, specific humidity and geopotential height of the NCEP / NCAR reanalysis were used and presented in table, graph and image forms, as a test of statistical significance. Mann Kendall and Student were applied. The results show that the seasonal variability of static energies for H.N and H.S presents maximum (minimum) values in the summer (winter) season. Positive trends are observed in the dry, wet and saturated static energy series, just as statistical tests indicate $99 \%$ significance $(\alpha=0.01)$ for trends. In the spatialization of trends on the Globe, there is an expressive coverage of areas with an average trend of -0.5 to $4.5 \times 10^{2}\left(\mathrm{~kJ} / \mathrm{m}^{2}\right) /$ year.
\end{abstract}

Keywords:: convection, Student's $t$, reanalysis, static energies.

\section{Introdução}

A Circulação Geral da Atmosfera se origina pela forma não homogênea da radiação recebida pela Terra, em que as regiões tropicais recebem maiores índices de radiação solar e, consequentemente, tornam-se mais aquecidas e úmidas em relação a regiões polares, cujo índice de radiação é menor e o aquecimento também, e características diferentes da superfície da Terra (solo nu, vegetação e água) afetam ainda essa distribuição de energia (Ayoade, 2012).

Trocas de massas de ar entre as regiões mais quentes e as mais frias, promove características térmicas diferentes

Autor de correspondência: Samyr Barata Chebly, samyrchebly@gmail.com. 
e contribui para o processo termodinâmico entre as energias estáticas seca, úmida e saturada na atmosfera em várias partes do globo, e consequentemente nas condições termodinâmicas da atmosfera. Como análises preliminares da energia estática podem ser encontrados em diferentes trabalhos (por exemplo, Mota e Nobre, 2006; Bengtsson et al., 2013).

As energias estáticas seca, úmida e saturada na atmosfera apresentam estruturas verticais semelhantes às apresentadas pelas temperaturas potencial, potencial equivalente e potencial de saturação, respectivamente (Nóbrega et al., 2006; AMS, 2018). Neste contexto, um fator importante diz respeito ao teor de água na atmosfera na forma de energia latente, que somada à energia estática seca é denominada de energia estática úmida. Quando ocorre condensação, parte dessa energia é liberada como calor sensível, enquanto que, o processo de evapotranspiração adiciona energia latente na atmosfera, mantendo em equilíbrio térmico o planeta (Peixoto e Oort, 1983; Allan, 2012).

Cavalcanti e Mariano (2016) observaram uma tendência de aumento do vapor d'água na atmosfera do globo com distribuição espacial não homogênea, mas com tendência positiva na maior parte do globo. O efeito estufa e as ações antrópica conforme Hardy (2003) estão diretamente ligados ao comportamento das energias estáticas na atmosfera.

Algumas pesquisas realizadas (Cavalcanti et al., 1996; Nóbrega et al., 2006; Bezerra e Cavalcanti, 2008; Bengtsson et al., 2013) sobre a energia estáticas relacionadas ao seu comportamento na atmosfera mostram que a energia solar e terrestre, o teor de vapor d'água, a interação oceano-atmosfera, os fenômenos meteorológicos e a Circulação Geral da Atmosfera através do transporte de calor e energia exercem uma influência na energia estática na atmosfera, tanto na variabilidade interanual como sazonal no planeta (Nóbrega et al., 2006). Para as regiões Norte e Nordeste do Brasil, alguns estudos mostraram que a umidade atmosférica provém da própria floresta Amazônica e, também, por advecção de vapor d'água do Oceano Atlântico. A disponibilidade de energia solar, a influência da Zona de Convergência Intertropical (ZCIT) e as anomalias de Temperatura da Superfície do mar (aTSM) dos Oceanos Pacifico e Atlântico modulam o comportamento das energias estáticas na atmosfera da região tropical e em especial do Norte e Nordeste do Brasil (Trenberth e Stepaniak, 2004; Nóbrega et al., 2006; Bezerra e Cavalcanti, 2008).

Bischoff e Schneider (2014) observaram que a ZCIT influência nas energias estáticas seca, úmida e saturada são meio do transporte meridional de energia, nas variações associadas com as mudanças energéticas, relacionadas com a diferença de temperatura inter-hemisférica e com o vapor d'água presente e na estrutura vertical da ZCIT e podendo apresentar uma atmosfera mais úmida em deter- minadas regiões do globo, assim como Bui et al.(2016) em seu estudo verificaram que o transporte vertical de umidade é sensível alta troposfera e baixa troposfera, movimento ascendente e movimento descendente, exportam e importam energia estática úmida positiva e negativa.

Os estudos abordando as energias estáticas na atmosfera apresentam períodos curtos de análises ou estão associados com determinados eventos que ocorrem em determinadas épocas do ano em algumas localidades do globo (Neelin e Held, 1987; Cavalcanti et al., 1996; Mota et al., 2006; Hill et al, 2017). Neste contexto, avaliar se existe uma tendência ao longo dos últimos trinta anos em relação às médias globais de energia estática seca e úmida para o globo, assim como, verificar o comportamento espacial dessas tendências parece salutar face as recentes discussões sobre tendências climáticas globais. Neste propósito, apresenta-se como objetivo geral analisar aspectos e tendências da energia estática da atmosfera global utilizando dados de reanálises do National Centers for Environmental Prediction/National Center for Atmospheric Research (NCEP/NCAR). Para tanto se faz necessário quantificar as energias estáticas seca, úmida e saturada para todo o globo; analisar as variabilidades sazonais, interanual da energia estática global; avaliar as tendências nas séries das médias mensais globais e a espacialização das tendências interdecenal globais.

\section{Material e Métodos}

\section{1. Área de estudo}

A área de estudo compreende todo globo (Fig. 1), entre as seguinte latitudes $90^{\circ} \mathrm{S}$ e $90^{\circ} \mathrm{N}$ e longitudes $180^{\circ} \mathrm{O}$ e $180^{\circ} \mathrm{E}$ para verificar as tendências médias globais e a espacialização das tendências interdecenal.

\subsection{Dados}

Os dados foram obtidos a partir de reanálises do NCEP/NCAR, que constituem-se numa base de informações de variáveis meteorológicas em pontos de grade provenientes de um sistema de assimilação de dados descritos por Kalnay et al. (1996) e Kistler et al. (2001). As observações assimiladas no modelo numérico refletem uma composição de observações por satélites; aviões; navios; boias oceânicas, além das observações convencionais. Neste contexto, são consideradas representativas das condições globais e tem sido utilizado com exito por alguns autores em estudos relacionados a energia estática na atmosfera (Nóbrega et al. 2006, Bezerra e Cavalcanti, 2008; Bischoff e Schneider, 2014; Bui et al., 2016). Portanto, se mostram adequadas para os propósitos estabelecidos para este trabalho.

Os dados utilizados correspondem às médias mensais do período de 1987 a 2016, cobrindo todo o globo e são espaçados em uma grade de $2,5^{\circ} \times 2,5^{\circ}$ latitude e 


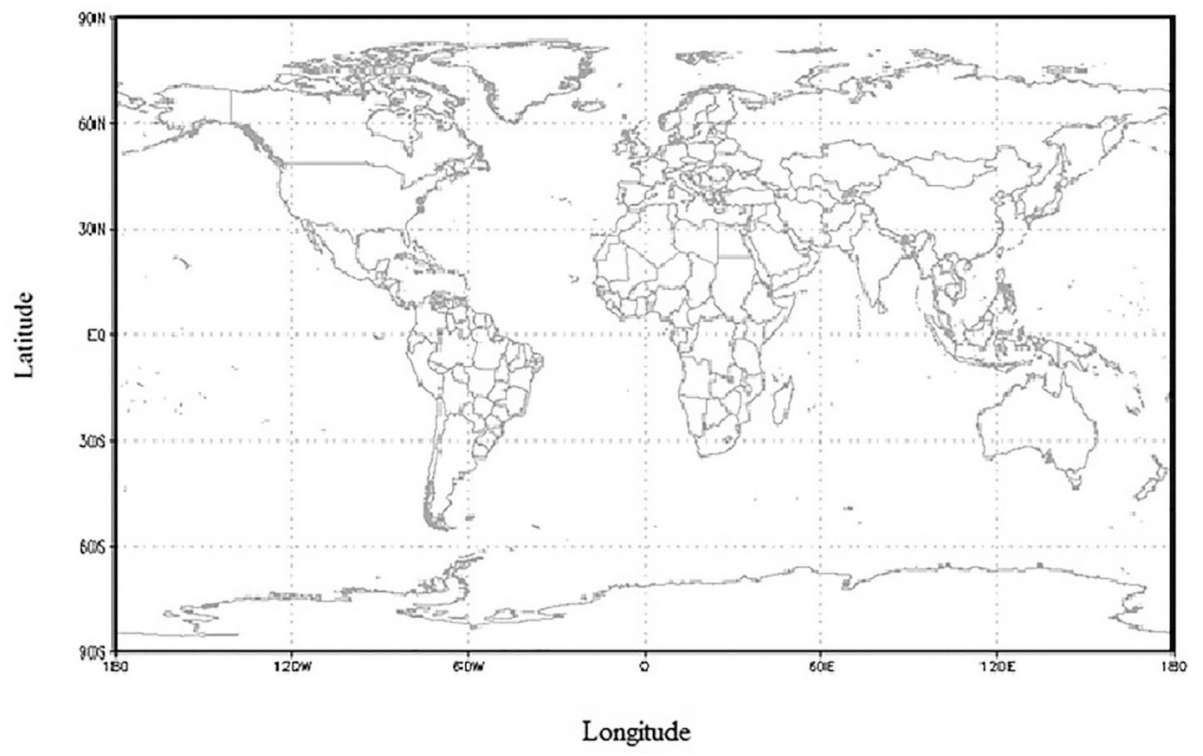

Figura 1 - Área de estudo: Globo Terrestre (Latitude: $90^{\circ} \mathrm{S}-90^{\circ} \mathrm{N}$, Longitude: $180^{\circ} \mathrm{O}-180^{\circ} \mathrm{E}$ ).

longitude. As variáveis que serão utilizadas são: pressão à superfície $(\mathrm{hPa})$, temperatura do ar $(K)$, altura geopotencial $(\mathrm{gpm})$ e umidade específica $(\mathrm{kg} / \mathrm{kg})$.

Foi utilizado um sistema de visualização e análise de dados em pontos de grade denominado Grid Analysis and Display System (GrADS). Este software é bastante utilizado nas tarefas de acesso, manipulação e visualização de dados geofísicos em geral, pois trabalha com matrizes de dados nos formatos BINÁRIO, GRIB, NetCDF ou HDFSDS, nos quais as variáveis podem possuir até 4 dimensões longitude, latitude, níveis verticais e tempo (Souza, 2004).

\subsection{Metodologia}

Para este estudo, foram utilizados dados mensais de pressão à superficie, temperatura do ar, geopotencial, umidade especifica de reanálies do NCEP/NCAR. A partir destes foram calculadas as energias estática seca, úmida e saturada, assim como, sua integração vertical.

Foram construidos gráficos para análise, referente a cada tipo de energia. Portanto, se adotou a seguite sequência: a) Variação sazonal e interanual: Médias para o Globo, Hemisfério Norte e Hemisfério Sul; (b)Tendências anual e espacialização: Avaliação de tendências nas séries anuais da média para o Globo, Hemisfério Norte, Hemisfério Sul e testes estatísticos $t$ de Student e Mann Kendall e em seguida feita uma espacialização da tendência para todo o Globo.

\subsubsection{Formulação teórica}

As equações de energia estática seca $(s)$, estática úmida $(h)$ e estática saturada $\left(h_{s}\right)$ são expressas em $\mathrm{kJ} / \mathrm{kg}$ segundo Nóbrega et al. (2006), Bezerra e Cavalcanti (2008), como:

$$
\begin{gathered}
s=c_{p} T+\varphi \\
h=s+L_{v} \\
h_{s}=s+L_{v} q_{s}
\end{gathered}
$$

em que $c_{p}$ é o calor específico à pressão constante

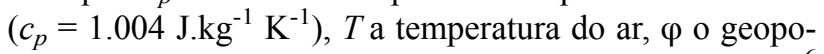
tencial, $L_{v}$ o calor latente de vaporização $\left(L_{v}=2,462 \times 10^{6}\right.$ $\mathrm{J} / \mathrm{kg}$ ), $q$ a umidade específica do ar e $q_{s}$ a umidade especifica do ar saturada. Portanto, $L_{v} q$ e $L_{v} q_{s}$ são as energias latentes úmida $(u)$ e saturada $\left(u_{s}\right)$.

A integração vertical dessas energias, expressas com a letras maiúsculas $I$, podem ser obtidas pelas expressões

$$
\begin{gathered}
I s=\frac{1}{g} \int_{p}^{p_{s}}\left(c_{p} T+\varphi\right) d p \\
I u=\frac{1}{g} \int_{p}^{p_{s}} L_{\nu} q d p \\
I u_{s}=\frac{1}{g} \int_{p}^{p_{s}} L_{v} q_{s} d p
\end{gathered}
$$

em que $p_{s}$ é a pressão à superficie e $p$ é a pressão no topo, tomada como $300 \mathrm{hPa}$, visto que, neste nível de pressão a umidade é praticamente nula.

Portanto, a soma da integração da energia estática seca com a integração da energia latente úmida, corresponde a integração da energia estatica úmida $(I h=I S+I u)$ e a soma da integração da energia estatica seca com a integração da energia latente saturada, corresponde a integração da energia estatica saturada $\left(I h_{s}=I s+I u_{s}\right)$. 


\subsubsection{Testes estatísticos}

Para isso foram utilizados os testes paramétricos e não paramétricos como o $t$ de Student e de Mann Kendall (MK), que em geral exigem amostras de dimensões de 30 anos ou inferior, dependendo da estacionariedade estatística, e são bastante empregados em pesquisas meteorológicas e em estudos com séries temporais climatológicas e hidrológicas, sendo recomendado pela Organização Meteorológica Mundial (OMM) para avaliar as tendências dos dados em séries temporais (Yue et al., 2002).

\subsubsection{Teste t de Student}

É um teste de hipótese que usa conceitos estatísticos para rejeitar ou não uma hipótese nula quando a estatística de teste $(t)$ segue uma distribuição $t$ de Student. Esse teste é muito utilizado nos estudos de meteorologia (Kousky e Kayano, 1994; Kayano e Kousky, 1996; Silva e Sousa, 2013).

Neste caso será aplicada a inclinação da linha de tendência pelo método da regressão linear do coeficiente de correlação $(r)$, com os valores observados das energias estáticas seca, umida e saturada. Aplicando o teste $t$ de Student com significância estatística de $99 \%(\alpha=0.01)$ e $95 \%(\alpha=0.05)$, com $(n-2)$ graus de liberdade, para $(n=30)$. É calculado da seguinte forma:

$$
\begin{gathered}
t=r \sqrt{\frac{n-2}{1-r^{2}}} \\
r_{c}=t_{c} / \sqrt{(n-2)+t_{c}^{2}}
\end{gathered}
$$

Os valores obtidos na tabela nessas condições foram de $t_{c}=2,763 ; t_{c}=2,048$, respectivamente. Obteve-se $r_{c}=0,463$ e $r_{c}=0,361$, portanto $r>r_{c}$ a hipotese nula é descartada caracterizando o valor significativo à $99 \%$ ou $95 \%$, respectivamente.

\subsubsection{Método de Mann-Kendall}

O Teste de Mann-Kendall (Mann, 1945; Kendall, 1975) é um método robusto, sequencial e não paramétrico utilizado para determinar se determinada série de dados possui uma tendência temporal de alteração estatisticamente significativa. Por tratar-se de um método não paramétrico, ele não requer distribuição normal dos dados (Yue et al., 2002). Outra vantagem deste método é o fato de ser pouco influenciado por mudanças abruptas ou séries não homogêneas (Zhang et al., 2009). No entanto, este método exige que os dados sejam independentes e aleatórios (Neeti e Eastman, 2011).

O método baseia-se em rejeitar ou não a hipótese nula $\left(H_{0}\right)$, de que não exista tendência na série de dados, adotando-se um nível de significância $(\alpha)$. O nível de significância pode ser interpretado como a probabilidade de cometer-se o erro de rejeitar a $H_{0}$ quando esta for verda- deira. Segundo Silva et al. (2010) a estatística S usada no teste de Mann-Kendall é dada por:

$$
S=\sum_{i=1}^{n} \sum_{j=i+1}^{N} \operatorname{sign}\left(x_{i}-x_{j}\right)
$$

em que $x_{j}$ são dados estimados da sequência de valores, $\mathrm{n}$ é o número de elementos da série temporal. Em que sign $\left(x_{i}\right.$ $\left.-x_{j}\right)$ é:

$$
\text { sinal }=\left\{\begin{array}{lll}
1 & \text { para } & x_{i}-x_{j}>0 \\
0 & \text { para } & x_{i}-x_{j}=0 \\
-1 & \text { para } & x_{i}-x_{j}<0
\end{array}\right.
$$

A estatistica $S$ tende a uma distribuição normal com média $E[S]$ e a variância $\operatorname{Var}[S]$, é dada por:

$$
E[S]=0
$$

$$
\operatorname{Var}(S)=\frac{1}{18}\left[n(n-1)(2 n+5)-\sum_{p=1}^{q} t_{p}\left(t_{p}-1\right)\left(2 t_{p}+5\right)\right]
$$

em que $n$ é o número de elementos da série, $t_{p}$ é o número de dados com valores iguais num certo grupo (pth) e $q$ é o número de grupos contendo valores iguais da série de dados no grupo $(p)$. O segundo termo representa um ajuste de dados

O teste estatístico parametrizado $(Z)$ é dado por:

$$
z=\left\{\begin{array}{lll}
\frac{S-1}{\sigma} & \text { se } & S>0 \\
0 & \text { se } & S=0 \\
\frac{S+1}{\sigma} & \text { se } & S<0
\end{array}\right.
$$

$\mathrm{O}$ valor de $Z$ resulta na presença de uma tendência estatisticamente significativa. Essa estatística é usada para testar a hipótese nula, ou seja, que nenhuma tendência existe. Um valor positivo de $Z$ indica tendência de aumento e quando negativa, indica tendência decrescente. Para testar a tendência crescente ou decrescente no nível de significância de $\alpha$, a hipótese nula é, então, rejeitada se o valor absoluto de $Z$ for maior que $Z_{1^{-\alpha / 2}}$, utilizando-se a tabela da distribuição normal cumulativa padrão. Em geral, usam-se os níveis de significância de $\alpha=0,01$ e 0,05 , que também serão aplicados neste estudo.

\section{Resultados e Discussão}

\subsection{Variação sazonal da energia estática}

As Figs. 2a-c apresentam as variações sazonais médias das energias estática seca (Fig. 2a), úmida (Fig. 2b) e 
saturada (Fig. 2c) para o H.N, H.S. e Globo, integrada verticalmente para os período de 1987 à 2016. É observado em todas as Figs. 2a-c que as energias estáticas seca, úmida e saturada apresentam um comportamento oposto no H.N. e H.S., obtendo valores máximos de energia estática seca, úmida e saturada no período de Julho-Agosto no H.N. e em Janeiro-Fevereiro para o H.S. na estação de Verão e de valores mínimos na estação de Inverno para Janeiro-Fevereiro no H.N. e em Julho-Agosto no H.S.

$\mathrm{Na}$ Fig. 2a mostram as médias mensais da energia estática seca que apresentaram máximos valores (mínimos valores) de $2896 \times 10^{3} \mathrm{~kJ} / \mathrm{m}^{2}$ e $2894 \times 10^{3} \mathrm{~kJ} / \mathrm{m}^{2}$ para o H.N e $2873 \times 10^{3} \mathrm{~kJ} / \mathrm{m}^{2}$ e $2874 \times 10^{3} \mathrm{~kJ} / \mathrm{m}^{2}$ no H.S $(2809$ $\times 10^{3} \mathrm{~kJ} / \mathrm{m}^{2}$ e $2810 \times 10^{3} \mathrm{~kJ} / \mathrm{m}^{2}$ no H.N. e de $2829 \times 10^{3}$ $\mathrm{kJ} / \mathrm{m}^{2}$ e $2828 \times 10^{3} \mathrm{~kJ} / \mathrm{m}^{2}$ no H.S) nos período de verão

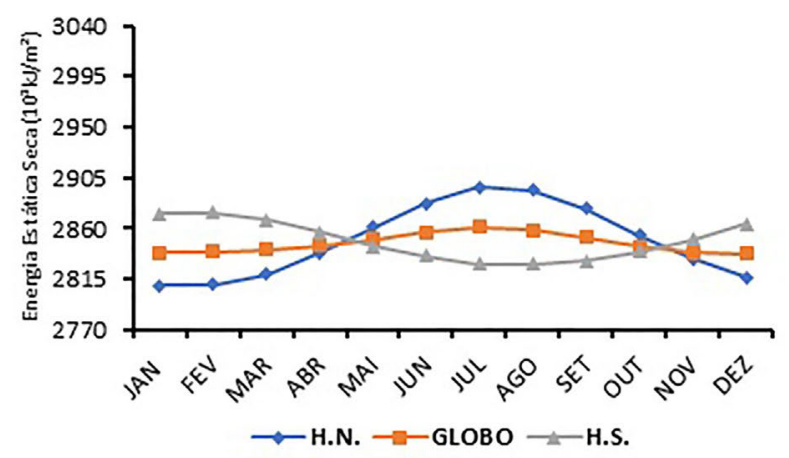

(A)

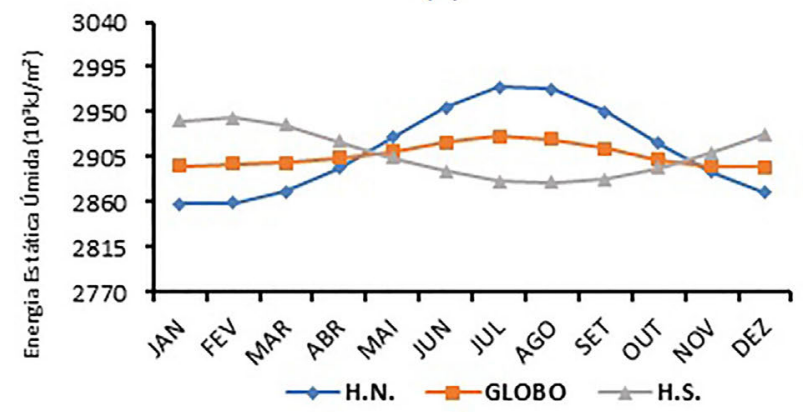

(B)

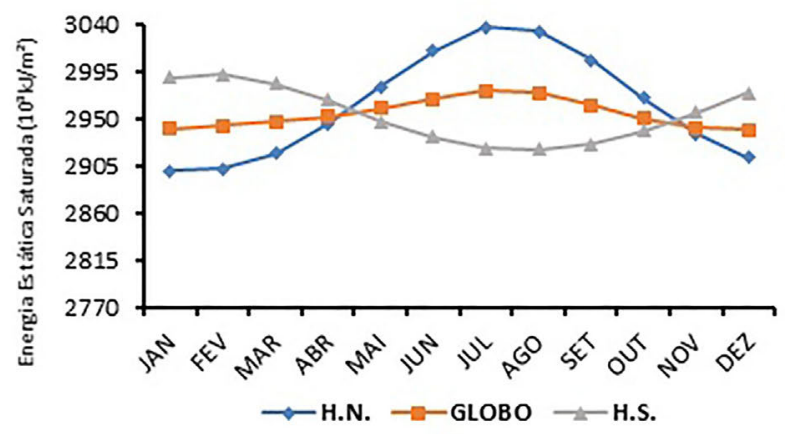

(C)

Figura 2 - Distribuição sazonal da média mensal das energias estáticas integradas verticalmente $\left(10^{3} \mathrm{~kJ} / \mathrm{m}^{2}\right)$ : (a) seca $\left(c_{p} T+\varphi\right)$, (b) úmida $(s$ $\left.+L_{v} q\right)$ e (c) saturada $\left(s+L_{v} q_{s}\right)$ para o H.N., H.S. e Globo, no período de 1987 a 2016. (inverno). A (Fig. 2b) apresenta as médias mensais da energia estática úmida com máximos valores (mínimos valores) de $2975 \times 10^{3} \mathrm{~kJ} / \mathrm{m}^{2}$ e $2973 \times 10^{3} \mathrm{~kJ} / \mathrm{m}^{2}$ no H.N e $2941 \times 10^{3} \mathrm{~kJ} / \mathrm{m}^{2}$ e $2944 \times 10^{3} \mathrm{~kJ} / \mathrm{m}^{2}$ para o H.S $(2858 \mathrm{x}$ $10^{3} \mathrm{~kJ} / \mathrm{m}^{2}$ e $2859 \times 10^{3} \mathrm{~kJ} / \mathrm{m}^{2}$ no H.N. e de $2881 \times 10^{3} \mathrm{~kJ} /$ $\mathrm{m}^{2}$ e $2879 \times 10^{3} \mathrm{~kJ} / \mathrm{m}^{2}$ no H.S) no verão (inverno). Para a (Fig. 2c) tem-se a média mensal da energia estática saturada com máximos valores (mínimos valores) de $3037 \mathrm{x}$ $10^{3} \mathrm{~kJ} / \mathrm{m}^{2}$ e $3033 \times 10^{3} \mathrm{~kJ} / \mathrm{m}^{2}$ no H.N e $2989 \times 10^{3} \mathrm{~kJ} / \mathrm{m}^{2} \mathrm{e}$ $2992 \times 10^{3} \mathrm{~kJ} / \mathrm{m}^{2}$ para o H.S $\left(2900 \times 10^{3} \mathrm{~kJ} / \mathrm{m}^{2}\right.$ e $2902 \mathrm{x}$ $10^{3} \mathrm{~kJ} / \mathrm{m}^{2}$ no H.N. e de $2922 \times 10^{3} \mathrm{~kJ} / \mathrm{m}^{2}$ e $2921 \times 10^{3} \mathrm{~kJ} /$ $\mathrm{m}^{2}$ no H.S) no verão (inverno), respectivamente. Observam-se que as médias das energias estáticas seca, úmida e saturada para o Globo apresentaram um comportamento mais similar e suavizado ao do H.N., com máximos valores no período de verão e mínimos valores no inverno do H.N.

A disponibilidade de energia solar sobre à superfície exerce influência no teor de vapor d'água na atmosfera que está diretamente relacionado com a temperatura do ar e consequentemente influenciando nas energias estáticas e na variação sazonal. Portanto, a média global (Figs. 2a-c) apresenta um comportamento sazonal mais parecido com o que ocorre para o H.N., visto que, o H.N. apresenta maior cobertura continental quando comparado ao H.S. Foram observados que os resultados se mostraram coerentes e similares com outros estudos (Nóbrega et al., 2006; Bezerra e Cavalcanti, 2008; Cavalcanti e Mariano, 2016), em que esse comportamento padrão pode mostrarse mais ou menos acentuado de ano a ano, sendo modulado por fenômenos de grande escala que vem estabelecer uma variabilidade interanual.

\subsection{Tendência anual e espacialização}

As Figs. 3a-i apresentam a série temporal das médias anuais para o período de 1987-2016 da energia estática seca (Figs. 3a-c), úmida (Figs. 3d-f) e saturada (Figs. 3g-i) integradas verticalmente, a partir de dados obtidos das reanálises do NCEP/NCAR, para o Hemisfério Norte, Hemisfério Sul e Globo, entre o período de 1987 à 2016.

Portanto, é observado que ocorre uma variabilidade da energia estática seca, úmida e saturada entre os anos estudados, assim como uma tendência positiva, que foi verificado através do coeficiente angular da linha de tendência. Essa tendencia é comprovada estatisticamente pelos métodos de Mann Kendall e $t$ de Student (Tabela 1). Para o H.N., H.S. e Globo (Figs. 3a-c) a energia estática seca apresenta valores entre 2835 a $2855 \times 10^{3}$ $\mathrm{kJ} / \mathrm{m}^{2}$, com valores máximos anuais de 2855,2852 e $2852 \times 10^{3} \mathrm{~kJ} / \mathrm{m}^{2}$ para os anos de 2016,2016 e $2016 \mathrm{e}$ mínimos de 2841,2842 e $2839 \times 10^{3} \mathrm{~kJ} / \mathrm{m}^{2}$ para os anos de 1993, 1999 e 1993 referente ao H.N., H.S. e Globo, respectivamente. É observado ainda que para a série temporal da média anual no H.N. (Fig. 3a) e Globo (Fig. 3c) apresentaram comportamentos similares e com 


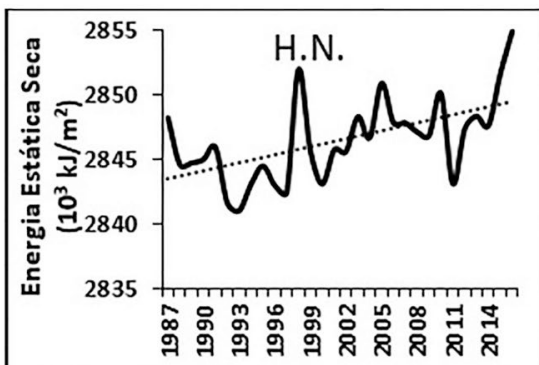

(A)

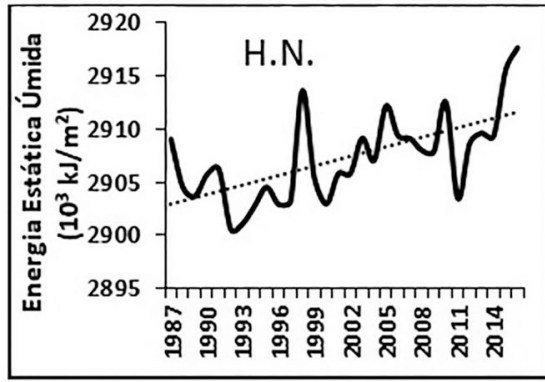

(D)

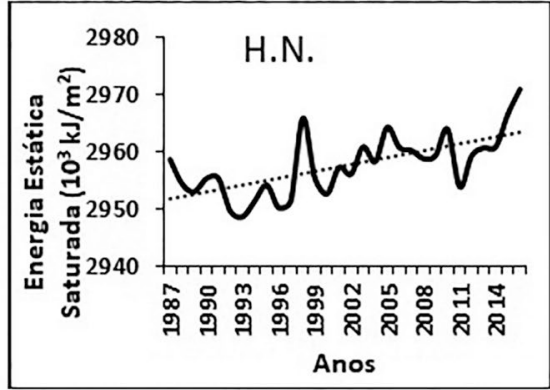

(G)

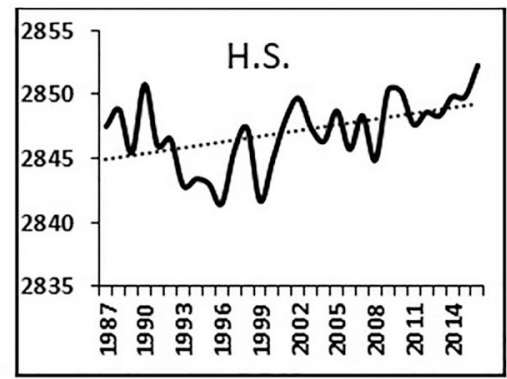

(B)

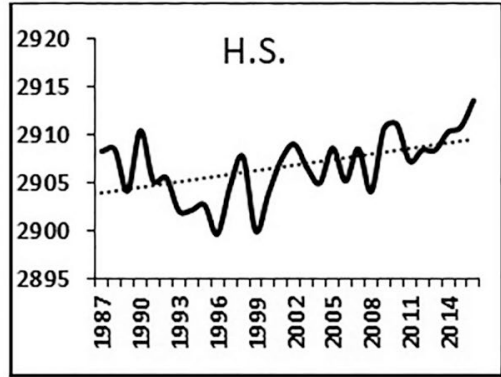

(E)

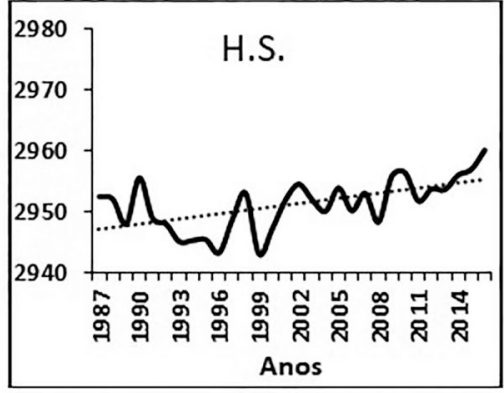

(H)

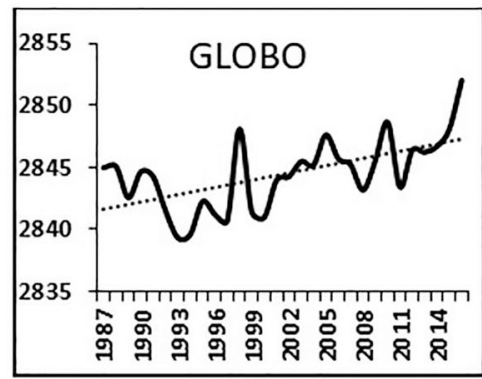

(C)

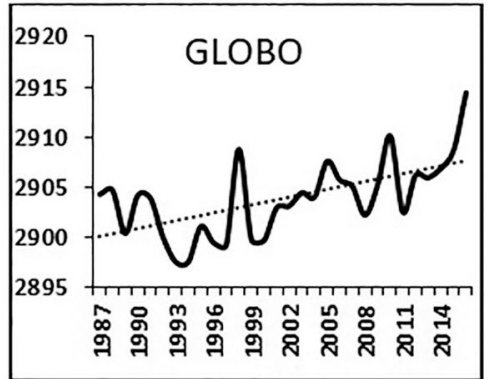

(F)

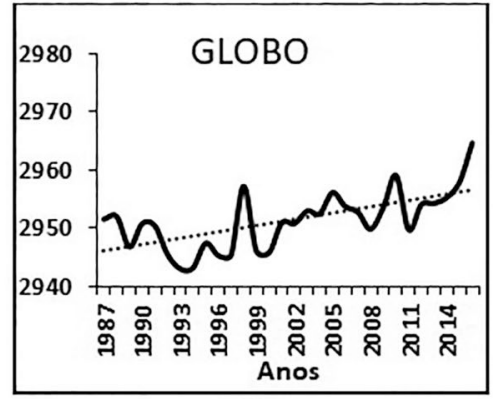

(I)

Figura 3 - Série temporal da média anual integrada verticalmente das energias estáticas seca $\left(c_{p} T+\varphi\right)$ (a, b e c), úmida $\left(s+L_{v} q\right)(\mathrm{d}$, e e f) e saturada $(s$ $\left.+L_{v} q_{s}\right)(\mathrm{g}, \mathrm{h}$ e i) para o Hemisfério Norte (H.N.), Hemisfério Sul (H.S.) e Globo para o período de 1987 a 2016.

valores superiores para o H.N. em relação ao Globo que segue a mesma tendência, sendo menos acentuada, enquanto para o H.S. (Fig. 3b) o comportamento da energia estática seca é distinta e de menores variações interanuais em relação ao H.N. e Globo.

Para as séries das médias anuais da energia estática úmida (Figs. 3d-f) observa-se comportamento similar ao discutido anteriormente para a energia estática seca, no entanto, diferem quanto a intensidade. Para o H.N., H.S. e Globo (Figs. 3d-f) a energia estática úmida apresenta variação média entre 2895 a $2920 \times 10^{3} \mathrm{~kJ} / \mathrm{m}^{2}$, com valores máximos de 2917,2913 e $2914 \times 10^{3} \mathrm{~kJ} / \mathrm{m}^{2}$ para os anos de 2016, 2016 e 2016 e mínimos de 2900, 2899 e 2897 x $10^{3} \mathrm{~kJ} / \mathrm{m}^{2}$ para os anos de 1992, 1996 e 1994 para o H.N., H.S. e Globo (Figs. 3d-f), respectivamente.

Observando a série da média anual da energia estática saturada, como esperado, apresenta mesmo comportamento ao discutido anteriormente. Neste caso os valores observados para o H.N., H.S e Globo (Figs. $3 \mathrm{~g}-\mathrm{i}$ ) variam entre 2940 a $2980 \times 10^{3} \mathrm{~kJ} / \mathrm{m}^{2}$, com valores máximos de
2971, 2960 e $2965 \times 10^{3} \mathrm{~kJ} / \mathrm{m}^{2}$ para os anos de 2016, 2016 e 2016 e mínimos de $2948,2943,2943 \times 10^{3} \mathrm{~kJ} / \mathrm{m}^{2}$ para os anos de 1993, 1999 e 1993 respectivamente. A ordem de grandeza dos valores obtidos para as energias estáticas seca, úmida e saturada são coerentes com valores obtidos em estudos realizados por Cavalcanti et al. (1996), Nóbrega et al., 2006 e Bezerra e Cavalcanti (2008).

A Tabela 1 apresenta parâmetros estatísticos obtidos pelos métodos de Mann-Kendall e $t$ de Student. Os dois casos apontam tendências positivas das energias estáticas seca, úmida e saturada para o H.N., H.S. e o Globo. As tendências das energias estáticas seca, úmida e saturada, segundo Mann-Kendall, foram 0,184; 0,288; 0,288 x $10^{3}$ $\mathrm{kJ} / \mathrm{m}^{2}$ ano para H.N.; 0,$164 ; 0,235 ; 0,291 \times 10^{3} \mathrm{~kJ} / \mathrm{m}^{2}$ ano para o H.S. e 0,$202 ; 0,276 ; 0,361 \times 10^{3} \mathrm{~kJ} / \mathrm{m}^{2}$ ano para o Globo, respectivamente. Esses valores apresentam significância estatística de $99 \%(\alpha=0,01)$ segundo MannKendall.

Para o método $t$ de Student, as tendências obtidas por regressão linear para as energias estáticas seca, úmida e 
Tabela 1 - Avaliação da tendencia e significância estatistica, através do método de Mann Kendell e $t$ de Student, para as séries anuais das Energias Estáticas Seca, Úmida e Saturada no período de 1987 a 2016.

\begin{tabular}{|c|c|c|c|c|}
\hline \multicolumn{5}{|c|}{ Energia Estática Seca } \\
\hline Método & Parâmetro & H.N. & H.S. & Globo \\
\hline \multirow{5}{*}{$\begin{array}{l}\text { Mann } \\
\text { Kendall }\end{array}$} & Inclinação $\left(10^{3} \mathrm{~kJ} / \mathrm{m}^{2}\right)$ ano & $0,184^{*}$ & $0,164^{*}$ & $0,202^{*}$ \\
\hline & t de Kendall & 0,431 & 0,359 & 0,441 \\
\hline & $S$ & 200,0 & 167,0 & 205,0 \\
\hline & $\operatorname{Var}(S)$ & 3460,667 & 0,000 & 0,000 \\
\hline & Valor - p & 0,000 & 0,002 & 0,000 \\
\hline \multirow[t]{3}{*}{$t$ de Student } & Inclinação $\left(10^{3} \mathrm{~kJ} / \mathrm{m}^{2}\right)$ ano & 0,212 & 0,148 & 0,197 \\
\hline & $\begin{array}{l}\text { Coeficiente de Correlação } \\
(r)\end{array}$ & $0,57^{*}$ & $0,47^{*}$ & $0,59^{*}$ \\
\hline & $b$ & 2843,2 & 2844,7 & 2841,4 \\
\hline \multicolumn{5}{|c|}{ Energia Estática Úmida } \\
\hline Método & Parâmetro & H.N. & H.S. & Globo \\
\hline \multirow{5}{*}{$\begin{array}{l}\text { Mann } \\
\text { Kendall }\end{array}$} & Inclinação $\left(10^{3} \mathrm{~kJ} / \mathrm{m}^{2}\right)$ ano & $0,288^{*}$ & $0,235^{*}$ & $0,276^{*}$ \\
\hline & t de Kendall & 0,478 & 0,372 & 0,428 \\
\hline & $S$ & 222,0 & 173,0 & 199,0 \\
\hline & $\operatorname{Var}(S)$ & 3460,667 & 0,000 & 0,000 \\
\hline & Valor - p & $<0,0001$ & 0,001 & 0,000 \\
\hline \multirow[t]{3}{*}{$t$ de Student } & Inclinação $\left(10^{3} \mathrm{~kJ} / \mathrm{m}^{2}\right)$ ano & 0,298 & 0,193 & 0,264 \\
\hline & $\begin{array}{l}\text { Coeficiente de Correlação } \\
(r)\end{array}$ & $0,63^{*}$ & $0,49^{*}$ & $0,60^{*}$ \\
\hline & $b$ & 2902,6 & 2903,7 & 2899,7 \\
\hline \multicolumn{5}{|c|}{ Energia Estática Saturada } \\
\hline Método & Parâmetro & H.N. & H.S. & Globo \\
\hline \multirow{6}{*}{$\begin{array}{l}\text { Mann } \\
\text { Kendall }\end{array}$} & Inclinação $\left(10^{3} \mathrm{~kJ} / \mathrm{m}^{2}\right)$ ano & $0,288^{*}$ & $0,291^{*}$ & $0,361^{*}$ \\
\hline & & & & \\
\hline & t de Kendall & 0,478 & 0,419 & 0,484 \\
\hline & $S$ & 222,0 & 195,0 & 225,0 \\
\hline & $\operatorname{Var}(S)$ & 3460,667 & 0,000 & 0,000 \\
\hline & Valor - p & $<0,0001$ & 0,000 & $\begin{array}{c}< \\
0,0001\end{array}$ \\
\hline \multirow[t]{3}{*}{$t$ de Student } & Inclinação $\left(10^{3} \mathrm{~kJ} / \mathrm{m}^{2}\right)$ ano & 0,409 & 0,279 & 0,366 \\
\hline & $\begin{array}{l}\text { Coeficiente de Correlação } \\
(r)\end{array}$ & $0,66^{*}$ & $0,56^{*}$ & $0,64^{*}$ \\
\hline & $b$ & 2951,3 & 2946,9 & 2945,6 \\
\hline
\end{tabular}

*Significância estatística para 99\% $(\alpha=0,01)$.

saturada, apresentam os seguintes valores 0,$272 ; 0,298$; $0,409 \times 10^{3} \mathrm{~kJ} / \mathrm{m}^{2}$ ano para H.N., 0,148; 0,$193 ; 0,279 \mathrm{x}$ $10^{3} \mathrm{~kJ} / \mathrm{m}^{2}$ ano para o H.S. e 0,$197 ; 0,264 ; 0,366 \times 10^{3}$ $\mathrm{kJ} / \mathrm{m}^{2}$ ano para o Globo, respectivamente. Os valores obtidos do coeficiente de correlação $(r)$ foram de $0,57,0,63$, 0,66 para o H.N; $0,47,0,49,0,56$ para o H.S. e $0,59,0,60$, 0,64 para o Globo e energias estáticas seca, úmida e saturada, respectivamente. Essas correlações, apresentam significância estatística de $99 \%(\alpha=0,01)$ segundo o teste $t$ de Student.
A Fig. 4a apresenta a espacialização da tendência da energia estática seca integrada verticalmente para o Globo, com os dados obtidos pelo NCEP/NCAR, que mostra grandes (menores) áreas com tendências positivas (negativas). É observado na faxia polar norte (acima de $60^{\circ} \mathrm{N}$ ) a presença de tendências positivas, atingindo valores de $4,5 \times 10^{2} \mathrm{~kJ} / \mathrm{m}^{2}$ ano e na faixa polar sul (acima de $60^{\circ} \mathrm{S}$ ) apresentou tendências positivas em grande parte da Antártica e tendências negativas na região do Oceano Pacifico Sul e Atlântico Sul, aproximandamente entre $\left(55^{\circ} \mathrm{S}-80^{\circ} \mathrm{S}\right.$ e $45^{\circ} \mathrm{W}-170^{\circ} \mathrm{E}$ ), como no Oceano Índico próximo $60^{\circ} \mathrm{S} \mathrm{e}$ $120^{\circ} \mathrm{E}$, com valores entre $-4,5 \times 10^{2} \mathrm{~kJ} / \mathrm{m}^{2}$ a $4,5 \mathrm{x}$ $10^{2} \mathrm{~kJ} / \mathrm{m}^{2}$ ano.

Para as latitudes médias norte $\left(30^{\circ}-60^{\circ} \mathrm{N}\right)$ observa grandes áreas com tendências positivas sobre o oeste e a leste do Oceano Pacífico Norte, que se expandem para América do Norte, a oeste e leste dos Estados Unidos, Norte do México, Golfo do México e a leste da Europa, Oriente Médio e regiões central, leste e oeste da Ásia com valores de 0,5 a $4,5 \times 10^{2} \mathrm{~kJ} / \mathrm{m}^{2}$ ano. Nas latitudes médias sul $\left(30^{\circ}-60^{\circ} \mathrm{S}\right)$ é observado que nos Oceanos Pacifico, Atlantico e Índico apresentaram tendências positivas e adentram as áreas da América do Sul e Oceânia, mais ao sul da Argentina e sul da Austrália. Para as tendências negativas, foram observados mais ao sul do continente africano, oeste do Chile e norte da Argentina com valores entre $-0,5 \mathrm{a}-1,0 \times 10^{2} \mathrm{~kJ} / \mathrm{m}^{2}$ ano. Nas áreas tropicais entre $\left(30^{\circ} \mathrm{S}-30^{\circ} \mathrm{N}\right)$ ocorreram tendências positivas sobre o Atlântico Tropical Norte e Sul, que se expandiram sobre a África e Oeste, Norte e Nordeste da América do Sul e mais ao Pacifico Tropical Norte e Sul obtendo valores de 0,5 a $3,0 \times 10^{2} \mathrm{~kJ} / \mathrm{m}^{2}$ ano. Em suma, tendências positivas da energia estática seca aponta uma tendência de aquecimento da atmosfera e resfriamento caso contrário, visto que, aquecimento implica em aumento do geopotencial e consequentimente aumento de energia estática seca $\left(c_{p} T+\varphi\right)$ e apresentando uma similaridade com estudos de Cavalcanti et al. (1996) e Bengtsson et al. (2013).

$\mathrm{Na}$ Fig. 4b que mostra a espacialização da tendência da energia estática úmida integrada verticalmente para o Globo, em que foram observados grandes áreas com tendências positivas nas faixas polares norte (sul) e latitudes médias norte (sul) bem mais extensas, com algumas similaridades ao da energia estática seca (Fig. 4a). Entretanto, as diferenças são observadas sobre a Ásia a oeste da China, a leste e oeste da Rússia, Índia, e expandindo-se mais a norte e leste dos Estados Unidos com valores entre 0,5 a $4,5 \times 10^{2} \mathrm{~kJ} / \mathrm{m}^{2}$ ano. Na faixa tropical que compreende aproximadamente entre $\left(30^{\circ} \mathrm{S}-30^{\circ} \mathrm{N}\right)$ é observado que as tendências positivas da energia estática úmida se expandem sobre toda a Oceânia, sudeste Asiático e em grande parte do Oceano Índico, com valores entre 1,0 e $4,5 \times 10^{2} \mathrm{~kJ} / \mathrm{m}^{2}$ ano, pois no Atlântico Tropical as tendências positivas abrangeram-se mais ao Atlântico Tropical 

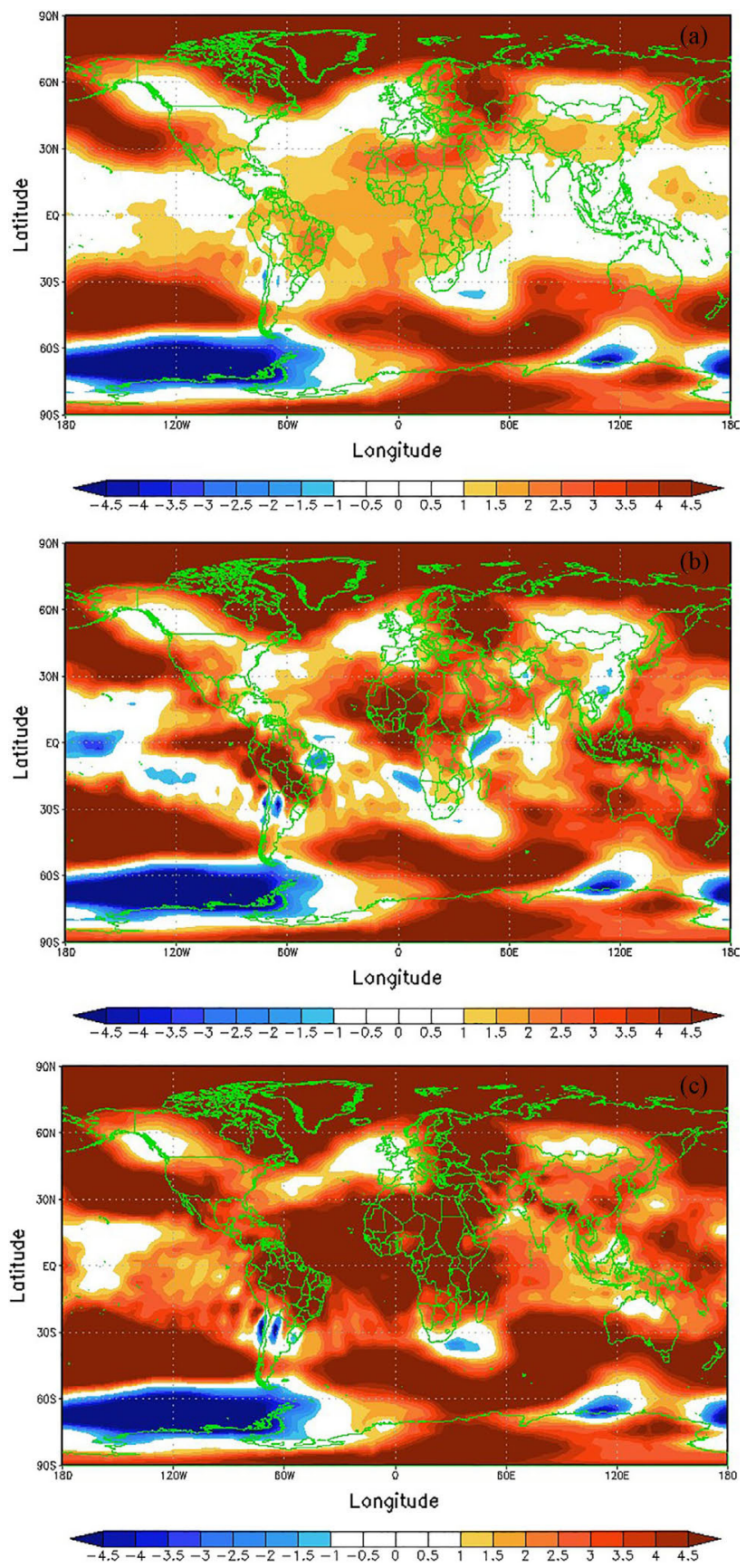

Figura 4 - Espacialização da tendência integrada verticalmente: (a) energia estática seca $\left(c_{p} T+\varphi\right)$, (b) úmida $\left(s+L_{v} q\right)$ e (c) saturada $\left(s+L_{v} q_{s}\right)$ $\left(10^{2} \mathrm{~kJ} / \mathrm{m}^{2}\right)$ ano para Globo.

Norte com expansão a Oeste e Leste da África, com valores de 1,0 a $4,5 \times 10^{2} \mathrm{~kJ} / \mathrm{m}^{2}$ ano, enquanto no Atlântico Tropical Sul tem-se menores áreas de tendência positivas, sendo mais visualizado a oeste e sudeste da América do Sul, pois para a região do Pacifico Equatorial nota-se que as tendências positivas ocorreram aproximadamente a $85^{\circ}-120^{\circ} \mathrm{W}$, com valores de 1,0 a $4,5 \times 10^{2} \mathrm{~kJ} / \mathrm{m}^{2}$ ano. Para as tendências negativas é observado que foram similares em determinadas áreas ao da (Fig. 4a), entretanto as diferenças ocorreram sobre a Região Tropical, visualizadas no NEB e Atlântico Equatorial com valores de -0,5 a $2,5 \mathrm{~kJ} / \mathrm{m}^{2}$ ano, no leste e sudeste da China entre $-0,5$ a $-1,0$ x $10^{2} \mathrm{~kJ} / \mathrm{m}^{2}$ ano, leste e sudoeste próximo a África de $-0,5$ a $-1,5 \times 10^{2} \mathrm{~kJ} / \mathrm{m}^{2}$ ano, proximo a região do Chaco argentino e leste do Chile e no Pacifico Equatorial, aproximadamente a $120^{\circ} \mathrm{W}, 150^{\circ}-180^{\circ} \mathrm{W}$ entre os valores de $-0,5$ a $-3,0 \times 10^{2} \mathrm{~kJ} / \mathrm{m}^{2}$ ano. Portanto, tendências positivas da energia estática úmida aponta, também, uma tendência de aquecimento da atmosfera e resfriamento caso contrário, visto que, aquecimento implica em aumento do teor de vapor d'água na atmosfera (Cavalcanti e Mariano, 2016) e consequentemente aumento de energia estática úmida $\left(s+L_{v} q\right)$, que favorece a convecção para a formação de nuvens com capacidade de precipitar.

Apresentando a espacialização da tendência da energia estática saturada integrada verticalmente para o globo (Fig. 4c), que mostram grandes áreas com tendências positivas que abrangem as faixas polares norte (sul) $90^{\circ}$ $60^{\circ} \mathrm{N}\left(90^{\circ}-60^{\circ} \mathrm{S}\right)$, latitude médias norte (sul) $60^{\circ}-30^{\circ} \mathrm{N}$ $\left(60^{\circ}-30^{\circ} \mathrm{S}\right)$ que abordaram aproximadamente mais os Oceanos Pacífico Norte (Sul) e Atlãntico Norte (Sul) com expansao leste e oeste, como ao sul do Oceano Índico e áreas da América do Norte, Europa, Ásia e Oceania com valores entre 0,5 e $4,5 \times 10^{2} \mathrm{~kJ} / \mathrm{m}^{2}$ ano. Para as regiões tropicais $\left(30^{\circ} \mathrm{S}-30^{\circ} \mathrm{N}\right)$ apresenta tendências positivas com valores de $4,5 \times 10^{2} \mathrm{~kJ} / \mathrm{m}^{2}$ ano sobre o Atlântico Tropical Norte e Sul, com expansão para África e em parte da América do Sul, enquanto que para as demais áreas obteve-se tendências positivas e negativas com valores entre $-0,5$ a $3,5 \times 10^{2} \mathrm{~kJ} / \mathrm{m}^{2}$ ano. Para as tendências negativas apresentadas o comportamento foi semelhantes nas faixas polares sul ao da (Figs. 4a-b) e distintos nas Latitude Médias Sul próximo da África mais ao sul e sobre o Sul do Brasil e oeste da Argentina e Chile com valores entre $-0,5 \mathrm{a}-4,5 \times 10^{2} \mathrm{~kJ} / \mathrm{m}^{2}$ ano. Em resumo, as tendências positivas da energia estática saturada mostram uma tendência de aquecimento da atmosfera, visto que, aquecimento implica em aumento do teor de vapor d'água na atmosfera (Cavalcanti e Mariano, 2016) e consequentemente aumento de energia estática saturada $\left(s+L_{v} q_{s}\right)$ em conjunto com a distribuição de energia estática úmida, favorecem processos de formação de nuvens convectivas (Nóbrega et al, 2006).

\section{Conclusão}

As tendências do comportamento das energias estáticas seca, úmida e saturada para o Globo, Hemisfério Norte e Hemisfério Sul no período de 30 anos de 19872016, e em face do que foi abordado e discutido, concluise que:

- A variação sazonal e interanual das energias estáticas integradas verticalmente para os H.N., H.S. e Globo foram máxima em julho-agosto no H.N. e janeiro-feve- 
reiro para o H.S. e para o Globo prevalece o comportamento similar ao H.N., sendo mais suavizada. Apresentando uma similaridade com

- As médias interanuais observadas das energias estáticas para o H.N., H.S. e Globo apresentaram um aumento de energia. Portanto, existe uma tendência de aquecimento e podendo estar relacionada com os fenômenos atmosféricos, ações antrópicas e mudanças climáticas, mencionadas na literatura.

- Os testes estatísticos de Mann Kendell apresentaram tendência significativas de $99 \%(\alpha=0,01)$ para o Globo, H.N, H.S.

- Para a espacialização no Global, obteve áreas de tendências positivas em praticamente todo o Globo Terrestre exceto em áreas menores do Hemisferio Sul. Expecula-se que pode estar relacionados as mudanças climáticas provocadas pelo efeito estufa mensionado na literatura.

Os resultados encontrados foram similares aos observados em ralação ao comportamento da variação sazonal (Nóbrega et al., 2006; Cavalcanti e Mariano, 2016), em tendências na série e espacialização (Bezerra e Cavalcanti, 2008; Bui et al., 2016; Cavalcanti e Mariano, 2016).

\section{Agradecimentos}

O presente trabalho foi realizado com o apoio da Coordenação de Aperfeiçoamento de Pessoal de Nível Superior Brasil (CAPES) - Código de Financiamento 001. Os autores agradecem ao Programa de Pós-Graduação em Meteorologia da Universidade Federal de Campina Grande (UFCG) e à CAPES, pela bolsa de Mestrado do primeiro autor e aos professores por todo o apoio.

\section{Referências}

ALLAN, R.P. The role of water vapour in Earth's energy flows. Surveys Geophysics, v. 33, n. 3-4, p. 557-564, 2012.

AMERICAN METEOROLOGICAL SOCIETY - AMS, Disponível em: https://www.ametsoc.org/index.cfm/ams/, acesso em: 08/06/2018.

AYOADE, J.O. Introdução à Climatologia para os Trópicos. Rio de Janeiro: Bertrand Brasil, 16 ${ }^{\mathrm{a}}$ ed, 2012.

BENGTSSON, L.; HODGES, K. I.; KOUMOUTSARIS, S.; ZAHN, M.; BERRISFORD, P. The changing energy balance of the polar regions in a warmer climate. Journal of Climate, v. 26, n. 10 p. 3112-3129, 2013.

BEZERRA, A.C.N.; CAVALCANTI, E.P. Energia estática sobre o norte e nordeste do Brasil relacionada com a temperatura da superfície do mar. Revista Brasileira de Meteorologia, v. 23, n. 2, p. 239-263, 2008.

BISCHOFF, T.; SCHNEIDER, T. Energetic constraints on the position of the intertropical convergence zone. Journal of Climate, v. 27, n. 13, p. 4937- 4951, 2014.

BUI, H.X.; YU, J-Y; CHOU, C. Impacts of vertical structure of large-scale vertical motion in tropical climate: Moist static energy framework. Journal of the Atmospheric Sciences, v. 73, n. 11, p. 4427-4437, 2016.

CAVALCANTI, E.P.; GOMES FILHO, M.F.; SILVA, A.R. Variabilidade da energia estática durante o experimento 'EMAS'. Atmosfera \& Água, v. 1, n. 1, p. 18-22, 1996.

CAVALCANTI, E.P.; MARIANO, E.B. Tendência do vapor d'água na atmosfera mediante dados do NCEP/NCAR. Revista Brasileira de Meteorologia, v. 31 n. 4, p. 564$569,2016$.

GNANADESIKAN, A.; RICHARD, D.; SLATER, R.D.; SWATHI, P.S.; VALLIS, G.K. The energetics of ocean heat transport. Journal of Climate, v. 18, p. 2604-2616, 2005.

HARDY, J.T. Climate Change. Cause, Effects and Solutions. Hoboken: John Wiley \& Sons, p. 260, 2003.

HILL, S.A.; MING, Y.; HELD, I.M.; ZHAO, M. A moist static energy budget-based analysis of the sahel rainfall response to uniform oceanic warming. Journal of Climate, v. 30, n. 15, p. 5637-5660, 2017.

KALNAY, E.; KANAMITSU, M.; KISTLER, R.; COLLINS W.; DEAVEN, D.; GANDIN, L.; IREDELL, M.; SAHA S.; WHITE G.; WOOLLEN J.; ZHU, Y.; CHELLIAH M.; EBISUZAKI, W.; HIGGINS, W.; JANOWIAK, J.; MO, K. C.; ROPELEWSKI, C.; WANG, J.; LEETMAA, A. REYNOLDS, R.; JENNE, R.; JOSEPH, D. The NCEP-NCAR 40-year reanalysis project. Bulletin of the American Meteorological Society, v. 77, n. 3, p. 437-471, 1996.

KAYANO, M.T.; KOUSKY, V.E. Tropical circulation variability with emphasis on interannual and intraseasonal time scales. Revista Brasileira de Meteorologia, v. 11, n. 1-2, p. 6-17, 1996.

KENDALL, M.G. Rank Correlation Methods. London: Charles Griffin, 1975.

KISTLER, R.; KALNAY, E.; COLLINS, W.; SAHA, S.; WHITE, G.; WOOLLEN, J.; CHELLIAH, M.; EBISUZAKI, W.; KANAMITSU, M.; KOUSKY, V.; DOOL, H.V.D.; JENNE, R.; FIORINO, M. The NCEP-NCAR 50-year reanalysis: Monthly means CD-ROM and Documentation. Bulletin of the American Meteorological Society, v. 82, n. 2, p. 247-267, 2001.

KOUSKY, V.E.; KAYANO, M.T. Principal modes of outgoing longwave radiation and 250-mb circulation for the South American sector. Journal of Climate, v. 7, n. 7, p. 11311143, 1994.

MANN, H.B. Nonparametric tests against trend. Econometrica, v. 13, p. 245-259, 1945.

MOTA, M.A.S.; NOBRE, C.A. Relação da variabilidade da energia potencial convectiva disponível (CAPE) com a precipitação e a alta da Bolívia durante a campanha "WetAMC/LBA". Revista Brasileira de Meteorologia, v. 21, n. 3, p. 344-355, 2006.

NEELIN, J.D.; HELD, I.M. Modeling tropical convergence based on the moist static energy budget. Monthly Weather Review, v. 115, n. 1, p. 3-12, 1987.

NEETI, N; EASTMAN, J.R. A contextual Mann-Kendall approach for the assessment of trend significance in image time series. Transactions in GIS, v. 15, n. 5, p. 599-611, 2011.

NÓBREGA, R.S.; SANTIAGO, G.A.C.F. Tendência de temperatura na superfície do mar nos oceanos Atlântico e Pací- 
fico e variabilidade de precipitação em pernambuco. Mercator, v. 13, n. 1, p. 107-118, 2014.

NÓBREGA, R.S.; SOUZA, E.P.; CAVALCANTI, E.P. Energia estática na atmosfera sobre o Nordeste do Brasil: Fluxo e variação sazonal de energia. Revista Brasileira de Engenharia Agrícola e Ambiental, v. 10, n. 1, p. 132-139, 2006.

PEIXOTO, J.P; OORT, A.H. The atmospheric branch of the hydrological cycle and climate. In: Variation in the Global Water. Dordrecht: D. Reidel Publishing Company, p. 5-65, 1983.

ROGERS, J.C.; SHENG-HUNG WANG, S.H.; COLEMAN, J.S.M. Evaluation of a long-term (1882-2005) equivalent temperature time series. Journal of Climate, v. 20, n. 17, p. 4476-4485, 2007.

SILVA, D.F.; SOUSA, A.B. Detecção de tendências climáticas no estado de Alagoas. Revista Brasileira de Geografia Física, v. 6, n. 3, p. 442-455, 2013.

SILVA, R.A.; SILVA, V.P.R.; CAVALCANTI, E.P.; SANTOS, D.N. Estudo da variabilidade da radiação solar no Nordeste do Brasil. Revista Brasileira de Engenharia Agrícola e Ambiental, v. 14, n. 5, p. 501-509, 2010.

SOBEL, A.; WHANG, S.; KIM, D. Moist static energy budget of the MJO during DYNAMO. Journal of the Atmospheric Sciences, v. 71, n. 11, p. 4276-4291, 2014.
SOUZA, E.B. GrADS - Fundamentos e Programação Básica. Curso de Extensão, Departamento de Meteorologia, Centro de Geociências, UFPA, 2004.

TAVARES, J.P.N.; MOTA, M.A.S. Condições termodinâmicas de eventos de precipitação extrema em Belém-PA durante a estação chuvosa. Revista Brasileira de Meteorologia, v. 27, n. 2, p. 207-218, 2012.

TRENBERTH, K.E.; STEPANIAK, D.P. The flow of energy through earth's climate system. Quarterly Journal of the Royal Meteorological Society, v. 130, n. 603, p. 26772701, 2004.

YUE, S.; PILON, P.; CAVADIAS, G. Power of the Mann-Kendall and Spearman's rho tests for detecting monotonic trends in hydrological series. Journal of Hydrology, v. 259, n. 1-4, p. 254-271, 2002.

ZHANG, W; YAN, Y; ZHENG, J; LI, L; DONG, X; CAI, H. Temporal and spatial variability of annual extreme water level in the Pearl River Delta region, China. Global and Planetary Change, v. 69, n. 1-2, p35-47, 2009.

License information: This is an open-access article distributed under the terms of the Creative Commons Attribution License (type CC-BY), which permits unrestricted use, distribution and reproduction in any medium, provided the original article is properly cited. 